\title{
Parameter Estimation for Rigid-Body Mass Moments of Inertia
}

\author{
S.A. McInerny \\ Department of Mechanical Engineering, The University of Alabama, P.O. Box 870276, Tuscaloosa, AL 35487-0276, USA
}

Two procedures that have the potential to reduce the time required to measure rigid-body mass moments of inertia by a factor of five, are presented. These procedures estimate the mass moments of inertia of a rigid-body from multiple-input, multiple-output (or multiple sets of single-input, multiple-output) vibration test data. The procedures are alternatives to the trifilar pendulum method of measuring the mass moments of inertia of a rigidbody. The first procedure described is the most direct, but requires that the forces on the test specimen at each of the mount attach points be directly measured using dynamic force gauges. The least squares method is then applied to an over determined set of equations involving only the six unique elements of the moment of inertia matrix. The second procedure, which does not require measurement of mount forces, is based on a sum of scalar equations (consisting of weighted vector norms) that eliminates the system damping matrix from consideration. The least squares method is then applied to an over determined set of equations for the six unique elements of the inertia matrix and the twenty-one unique elements of the system stiffness matrix.

\section{NOMENCLATURE}

$\left\{b_{j}\right\}$ - Unit direction vector for applied force " $j$ " and resultant moments: $\left\{b_{j}\right\} \equiv\left[\begin{array}{c}I \\ G_{j}\end{array}\right]\left\{e_{j}\right\}$.

c.g. - Center of gravity.

$\left[D_{\text {eff }}\right]$ - Effective system damping matrix. This matrix represents a viscous damping model of the system losses. It is a real, symmetric, $6 \times 6$ matrix.

$\left\{e_{j}\right\}$ - Unit direction vector for applied force " $j$ ".

$\tilde{F}_{j}(\omega)$ - Fourier transform of the magnitude of applied force " $j$ ".

$\left[G_{i}\right]$ - Matrix whose elements are components of the distance vector $\left\{r_{i}\right\}$. This matrix is used to represent cross-products, e.g., $\left\{r_{i}\right\} \times\{F\}$ in matrix multiplication form.

$[\tilde{H}]_{j}$ - Matrix whose columns are the values of the transfer functions between measured accelerations and the " $j$ "th applied force; each column corresponds to a single frequency.

$[\tilde{H}]$ - Matrix of transfer functions between measured accelerations and all of the applied forces, a $12 \times 3 N$ matrix (12 accelerations, 3 applied forces, $N$ frequencies). See Eq. (13).

$\left\{\tilde{H}_{c}\left(\omega_{k}\right)\right\}$ - Vector of the transfer functions between the rigid-body accelerations and the applied forces, a $6 \times 1$ vector.

$\left[\tilde{H}_{c}\right]$ - Matrix of the transfer functions between the rigidbody accelerations and all of the applied forces, a $6 \times 3 N$ matrix ( 3 applied forces, $N$ frequencies). See Eq. (14b).

$\left\{H_{R}\right\},\left\{H_{l}\right\}$ - The real and imaginary parts of vector $\left\{\tilde{H}_{c}\left(\omega_{k}\right)\right\}$. See Eq. (19).

$\left[\tilde{H}_{F}\right]$ - Matrix of the transfer functions between the reaction forces at the mounts and all of the applied forces, a $9 \times 3 N$ matrix ( 3 mounts, 3 applied forces, $N$ frequencies). See Eq. (15b).

$\left\{\tilde{H}_{F}(\bullet, n)\right\}$ - Column " $n$ " of $\left[\tilde{H}_{F}\right]$. See Eq. (15c).
$[I]$ — Identity matrix, here a $3 \times 3$ matrix.

$J_{i j}$ - Elements of the $3 \times 3$ symmetric, i.e. $J_{i j}=J_{j i}$, mass moment of inertia matrix.

$J_{k}$ - Principal moment of inertia, $k=1-3$.

$[J]$ - Rigid-body mass moment of inertia matrix, a $3 \times 3$ matrix.

$\left[K_{\text {eff }}\right],[M]$ - System stiffness and equivalent inertia matrices, respectively. Both are real symmetric $6 \times 6$ matrices.

$[Q]$ - Orthogonal matrix whose columns are the principal direction vectors corresponding to the principal moments of inertia, a $3 \times 3$ matrix.

$\left\{q_{k}\right\}$ - Principal direction vector corresponding to $J_{k}$, a $3 \times 1$ vector.

$\left\{r_{a}\right\}$ - Location of accelerometer in the body-fixed coordinate system, a $3 \times 1$ vector. Four locations, denoted by subscripts $a, b, c$ and $d$, are assumed.

$\left\{r_{i}\right\}$ - Location of mounting point " $i$ " ( $i=1$ to 3$)$ in bodyfixed coordinate system, a $3 \times 1$ vector.

$\left\{R_{c}\right\}$ - Vector of the translational and rotational displacements of the rigid-body relative to the inertial coordinate system, a $6 \times 1$ vector.

$\{T\}-$ Moments generated by forces on the rigid-body.

$[\Omega],\left[\Omega^{2}\right]$ - Diagonal $N \times N$ matrices whose elements are $\omega_{k}$ and $\omega_{k}^{2}$, respectively.

\section{INTRODUCTION}

Commonly used experimental methods for determining mass moments of inertia involve suspension of the body and measurement of its period of oscillation about an axis through its centroid..$^{1-5}$ The Air Force uses a torsional pendulum to measure human body mass moments of inertia about three orthogonal axes, for use in the design of military aircraft ejection seats. ${ }^{6}$ Automotive manufacturers require principal moments of inertia for motor-gearbox assemblies, and their direction vectors, in order to analyze the dynamic behavior of their vehicles.

The principal moments of inertia $J_{1}, J_{2}$ and $J_{3}$ and their direction vectors $\left\{q_{1}\right\},\left\{q_{2}\right\}$ and $\left\{q_{3}\right\}$, are determined from 